THEORIA ET HISTORIA SCIENTIARUM, VOL. VI, N²

Ed. Nicolas Copernicus University 2002

Christine Parker-Jones, Rachel Pilkington

\title{
Can Concept Maps Support Medical Students Learning From Simulation?
}

\begin{abstract}
Medical students need to acquire critical thinking skills to support diagnostic reasoning. Simulations can be particularly useful in providing a meaningful context in which students can both acquire conceptual models of complex systems and practise diagnostic reasoning. However, engagement with simulations can be shallow and their foil potential for learning may not be realised unless students are supported with appropriate active tasks and scenarios. This paper examines the use of Concept Maps as an aid to learning whilst interacting with a simulation of calcium metabolism. The paper reviews the ways in which Concept Maps might plausibly assist students engaged in the problem-solving and planning activities associated with diagnosis and treatment of calcium imbalance. A study is reported which indicates that Concept Maps can be an aid to learning both alongside the use of simulation and in their own right. Concept Maps also provide useful insights into the completeness and consistency of students' mental models following interaction with a simulation model.
\end{abstract}

\section{Concept Maps}

\section{Uses of Concept Maps}

This paper examines the usefulness of Concept Maps as a support tool for medical students when learning from simulations. Concept Maps have been used in a range of contexts to provide a visual representation of knowledge structures and argument forms (Gaines and Shaw, 1995). As such, they complement natural language as a means of communicating knowledge. Concept Mapping has also been used in a variety of educational settings to probe and reveal cognitive 
structures (e.g. West, Fensham and Garrard, 1982) and to provide a forum for sharing, exchanging and generating different and collective points of view. Novak and Gowin (1984) identify six educational applications of Concept Mapping:

Exploration of what a student already knows,

Road mapping a learning route,

Summarising - extracting meaning from textbooks,

Extracting meanings from laboratory, studio and/or field studies.

Reading articles

Writing - Planning a paper or exposition

The technique of Concept Mapping is usually associated with Novak (Novak and Godwin, 1984) and based on work by Ausubel. The term "Concept Map" generally refers to two-dimensional diagrams (See Figure 1) used to illustrate relationships between ideas within a particular domain. These ideas, objects or concepts are defined and described through propositions, frequently represented by labelled ellipses, joined together by arcs or lines to indicate the relationships between them. Labelling of the relationships is optional, but it is generally accepted that more information can be elicited from the Concept Maps if they are fully labelled (Stewart, 1979). Multiple linkages depict richer or more complex sets of inter-related concepts. Concept Maps are said to be an explicit and external representation of our internal integrated and hierarchical knowledge networks. The act of Concept Mapping is thought to help the learner activate meaningful learning by explicitly relating what they already know to new information.

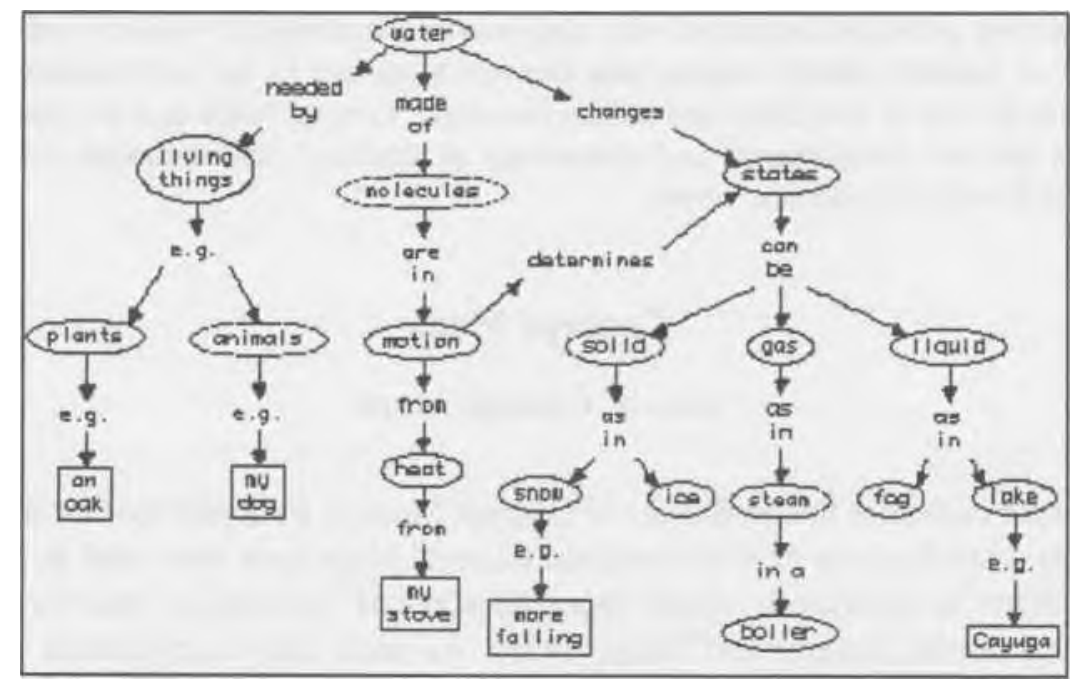

Figure 1. Concept Map of student's knowledge (after Novak and Gowin, 1984) 
According to Ausubel's assimilation theory of cognitive learning (Ausubel, 1968), learning becomes meaningful when it occurs in the context of the learner's prior knowledge. The acquisition of new knowledge is accomplished through the learning of new concepts and propositions, and to be meaningful, the learner must actively link this information to their existing, related knowledge structures. This causes a reformulation or Integrative Reconciliation of previously learned concepts (Ausubel, Novak and Hanesian, 1978). If the aim is to make instruction more effective, then tools such as Concept Maps that allow us to see the learner's initial knowledge state are critical. However, Concept Maps are not regarded as a diagnostic tool alone, they are thought to actively aid the learning process when constructed by students as part of the learning activity.

It is thought that Concept Maps can be an effective aid to learning because they encourage creativity, explication and reflection. The construction of Concept Maps encourages students to reflect on what to include or exclude whilst the linking and labelling of objects is a means of articulating their model of the world. Reflection can be 'on action', which occurs either following or by interrupting the activity, or it can be 'in action', which occurs during the activity by thinking about how to re-shape the activity while it is under way (problem solving).

The use of Concept Mapping techniques in the latter way is consistent with situated cognition (Brown et al, 1989), as the concepts are both situated and progressively developed through activity. In this activity there are two levels of engagement, the first is the operational level where nodes are created, positioned and moved whilst the second, the cognitive level involves thinking. Thinking precedes operation, but as the operations will precipitate thinking, the process is cyclic. Engagement is thus a cognitively active process that supports the manipulation of knowledge artefacts, ideas and concepts.

It is often possible for one representation to be transformed or translated into another representation, what is less certain is what distinguishes a Concept Map from related types of entity-relation diagrams, or how these differences affect learning. There is a raft of different diagrammatic methods for illustrating knowledge about a particular domain, the concepts involved and the relationships between them. Indeed, the same information can be illustrated in a number of ways (text, table, graphic), and the degree to which the construction of a diagram is constrained determines its content and is likely also to affect its properties for learning and problem-solving. There is evidence from related studies adopting a number of alternative external representations that the form of representation chosen by the student or imposed by the cognitive tool affects the ease of problem solving (Cox and Bma 1995; Suthers, 1999). In an analysis of students' work- scratchings Cox and Bma concluded that selecting an appropriate representation, one which is capable of representing the semantics of the problem, is difficult 
for students and that selecting an inappropriate representation could affect problem solving success. Likewise, Suthers (1999) suggests that the type of representation used in computer based systems could produce a representation bias, constraining the knowledge that can be expressed and it salience during problem-solving.

Glynn (1997) advocates the drawing of mental models as a method of allowing teachers to examine students' mental models of science concepts, and argues that these drawings should not be confused with other teaching strategies, such as concept mapping. However, he goes on to argue that the students should be encouraged to clarify their drawings by labelling features, drawing arrows, and including as many relevant details as possible - comments which are very similar to those made by Stewart about concept mapping.

While Novak refers to hierarchical maps other authors favour the use of central concepts. With this approach, it is possible for the focus to change - another concept becomes the main concept and the links radiate from it. If this occurs on a number of occasions, it is not always possible to determine which was the original starting point. The types of relation to be expressed may also differ widely. Hierarchical maps will tend to make use of „,is a”, „has” and „,contains” links, whereas other types of map may represent linear or cyclic causal processes making use of various causal influence relations.

Common to all these types of Concept Map, the individual is able to construct and manipulate the concept representations and engage in the processes of checking, resolving and confirming understandings. In this way, McAleese (1994) claims maps are part of the learning process, auto-monitoring, rather than a manifestation of learning itself. Automonitoring, or the monitoring of one's own process of learning, is pivotal in understanding the operation of Concept Maps and involves a series of stages:

Acknowledgement: the state of initial awareness where the learner thinks about some concept.

Representation: the formal activity such as creating a concept label.

Viewing: the core activity of being perceptually aware of the entities in the learning area.

Checking: the concept labels are compared, and the learner attempts to make sense of the nodes and links.

Resolution: any conflicts that occur in the labelling or linking are examined and redressed.

Confirming: the agreement that the representation is what was intended and has no inconsistencies.

Traditionally Concept Maps have been drawn with paper and pencil, but as education has become more computer-based in recent years, software has been developed to produce Concept Maps. Indeed, Jonassen and Mana (1994) include Concept Maps in their list of Cognitive tools or Mindtools - computer-based 
tools and learning environments that have been adapted or developed to support critical thinking in the form of knowledge representations. Cognitive tools are both mental and computational devices that support, guide, and extend the thinking processes of their users (Derry, 1990). They require the student to think mindfully in order to use the application to represent what they know, and it is this engagement which enables the students to learn and retain knowledge.

A Concept Mapping Tool as an exemplar Cognitive Tool actively engages the student in the generative processing of information, whereby deeper understanding results from activating appropriate mental models, and using them to interpret new information. This new information is assimilated back into those models, which are reorganised in light of the newly interpreted information, and these aggrandised models are used to explain, interpret or infer new knowledge. In this way, by constructing a Cognitive Map, learners engage in knowledge construction rather than knowledge reproduction.

It is coctended computers support reflective thinking when they enable users to compose new knowledge by adding new representations, modifying old ones, and comparing the two. These are the purposes of Concept Maps. To summarise Concept Maps are said to be:

- A learner centred tool for fostering active engagement and construction.

- A performance indicator, both before and after a learning experience, and as such can be used as a method of assessment.

- A method of envisioning, which in turn helps abstract reasoning.

- A tool for focusing the student on the area of interest, acting as a prompt for recalling information.

- A form of self-explanation that help students to identify and fill in the gaps in their knowledge.

- Provide a social focus for debate. The tutor or tutoring system can highlight these gaps in knowledge and undertake remedial action or influence the path that a student takes through the resource.

- Provide an alternative more systematic and abstract representation helping to bridge between the development of concrete and abstract understanding.

\section{Concept Map Construction}

There are a number of ways in which Concept Maps can be constructed; all of which have their advantages and disadvantages, and roles to play in the different learning environments. The simplest form of construction is from concept names given to the respondent, either as labels to be arranged in a suitable order or as a list to be built up as an increasingly specific map. These methods provide strong clues to the students about what to include and are probably not suitable 
for use in higher education: the level of priming is too high. However, they might be an appropriate method to study how students viewed the relationships between a fixed group of entities. Alternatively, Students may be required to identify concepts to include from a larger set that contained some foils or, the student may be given only the key concept and rules for drawing a Concept Map, including the requirement to label all relationships. This involves the student in much more work, by requiring that they generate the entities and relations to include for themselves. This method reveals the propositions that the learner sees as linking the concepts. However, although there is a basic assumption that Concept Maps reveal part of the student's thinking process about a particular topic, it is unreasonable to assume that a Concept Map is a representation of how concepts are stored in the student's mind.

\section{Scoring Concept Maps}

If Concept Maps can be used to indicate a student's knowledge it seems feasible that this can be quantified in some way to give an assessment of learning resulting from interactions with learning materials. Indeed, while Novak and Gowin (1984) were primarily interested in qualitative changes in the representation of children's conceptual framework as a result of instruction over time, they went on to develop rules for scoring Concept Maps in response to the pressure to be able to make quantified claims. The rules they developed were applicable to hierarchical maps, and scored students along five different axes, included:

- Breadth of knowledge - A score for all valid relationships, with the possibility of subtracting wrong or ambiguous concept links. Valid crosslinks could be scored under this rule by the degree of integration.

- Depth of knowledge - A weighted score for each valid level of the hierarchy. As valid hierarchies' signal progressive differentiation and integrative reconciliation of concept meanings, it seems reasonable to weight the number of levels above the number of relationships. Scoring levels of hierarchy in asymmetrical maps is a potential problem, but Novak suggests that the number of valid hierarchies in the most branched segment of the map be counted, giving an indication of the depth of knowledge.

- Degree of integration - A score of cross-links that show a valid relationship between two distinct segments of the concept hierarchy. These signal possibly important integrative reconciliation. As such they may be better indicators of meaningful learning than are hierarchical levels. It is a subjective decision whether a cross-link represents substantial integrative reconciliation of two sets of concepts. 
- Specific examples - Specific examples are useful to check that the student knows what kind of event or object is designated by the concept label.

- Expert or complete view - A comparison with a criterion Concept Map with a score based on the percentage of congruency. The criterion map could be an amalgam of all the students map, or it could be an ideal map, as constructed by one or more experts. Wallace and Mintses (1990) modified Novak's scoring system in their study of Concept Maps as vehicles for documenting and exploring conceptual changes in biology. They produced a specific scoring system including Relationships, Hierarchies, Degree of Branching, Cross-linking and General to specific examples. This work is one of the few examples of attempts to use Concept Mapping to evaluate learning. The main difference between their approach and Novak's was the lack of comparison with a criterion map. This has the disadvantage that if the students know the rules by which it is marked, it is easy to construct maps which would score more highly while not being more valid in terms of domain content, or showing evidence of deeper understanding. Their experiment sought to assess the extent to which changes in students' cognitive structures were reflected in their Concept Maps. The experimental group studied a CAI program on "Life Zones in the Ocean" while the control group received an equivalent exposure to an unrelated topic "Body defences". The pre- and post- tests were identical. As expected, the students' scores on the "Life Zones" revealed small but significant differences favouring the experimental group. The analysis of the Concept Maps was split into their scores and the number of critical concepts and propositions depicted.

While the analysis of the scores revealed significant differences in all five scoring categories, the novel aspects in this paper were the examination of critical concepts and propositions, and the use of follow-up interview to verify the results of the scoring systems. The critical concepts and propositions were identified and agreed by a panel of three outside educators. After viewing the program they produced their own Concept Maps. The experimental group demonstrated a larger number of critical concepts and propositions. Post hoc interviews were conducted with the five students from the experimental group who had scored highest and lowest. These revealed a potentially strong relationship between the complexity of cognitive structure as revealed in Concept Maps and the extent of biologically meaningful knowledge possessed by the learner.

Stuart (1985) also uses Concept Maps as a form of assessment and discusses the problems of scoring maps objectively. Though Novak advocates a hierarchical arrangement starting with the general, most inclusive concept at the top, Stuart (1983) favours a central position for the most general approach and other concepts radiating outward. Her conclusions are that reliance on numerical scores with the different components either aggregated or treated separately is to risk missing 
research data of great value as well as diagnostic data used to help the pupil. The components of the maps scored are not always independent and do not always have a strong relationship with performance. She argues against combining the component scores on the grounds that reliability was lost. It was also found that deleting one component score from the calculation made considerable difference to the level of reliability. When the component scores were kept separate it was possible to detect differences in the level of complexity of the Concept Maps of learner in a pre-test/ post-test situation and between different learners.

In the medical domain we are interested in students' causal models. Diagnosis and treatment is a combination of pattern matching and working from first principles. Diagnostic hypotheses need to be distinguished between by inquiry in order to make a diagnostic decision. Thus the clinician needs to be able to rely on causal reasoning where the pattern-matching approach presents more than one possibility and they are unsure of the diagnosis. When treating a disorder they have to be able to predict the effects of their treatment both at a primary level and any side effects. Concept Maps are one method of trying to access students' mental models concerning underlying causal relationships. We wanted to investigate whether students could produce a concept map representing these causal relationships, and we wanted to know whether their ability to produce Maps accurately representing the direction of such relationships and their positive or negative affect, would relate to success on diagnostic problems. Related research (Paton, 1996), suggests that students able to produce such an external representation ought to benefit from having such a cognitive bridge when reasoning about (making inferences from) the biological system to the diagnostic problems. This would however depend on students not only being able to represent these relationships appropriately but also being able to infer appropriately from them to draw conclusions about diagnosis and treatment.

\section{The Medical Context}

In our chosen instructional domain the main learning goals are to develop conceptual understanding and diagnostic reasoning skills amongst medical students. Two computerbased simulation models were used to provide the learning context for the reported study - one of which models the regulation of calcium levels within the body and the other models the activity of the thyroid gland. Both models are similar but for the sake of clarity, the examples used in this paper are all taken from the calcium simulation, which is the more complex system. In this model, dietary calcium is absorbed into the plasma (the liquid fraction of the blood), through the gut wall. In controlling the level of plasma 
calcium, the key point is that the system is tightly self-regulatory: It is the level of calcium itself that controls how much calcium is absorbed or excreted. The mechanism involves a negative feedback loop acting on the parathyroid. The parathyroid secretes PTH (parathyroid hormone), and the effect of this is to increase plasma calcium in at least three ways, one of these is the stimulation of calcitriol production. This, in turn, increases the amount of calcium absorbed from the gut. However, this also requires adequate supplies of vitamin D3.

Imbalance or malfunction can, thus, result from a number of causes. Clearly, this is a complex system (figure 8 shows just a small part of the calcium model developed using Stella, a computer based modelling package). Clearly, medical students can be expected to find difficulty in diagnosing faults and making corrective interventions unless they have a good grasp of the basic functioning of this system. It is thus, important that students become aware of these difficulties in diagnosis, learning how to differentiate between possible causes and to apply sensible treatment regimes.

One approach to teaching students these complexities would be to involve them directly in simulation modelling using the modelling software. This software provides abstract causal and flow relations and a number of different entities that can be used to represent variables affecting the flow of substances around the system. By instantiating the model with initial values and "running" it the modeller derives output data used to refine the behaviour of the model through cyclic adjustment of the model's parameters. However the complexity of the modelling software and the model to be constructed would mean that students would spend their full chemical pathology placement learning to model one system when there is need to grasp several such systems in the available time. The solution would seem to lie with using pre-built simulation models to simulate cases that the student must diagnose and treat, providing the bridge between textbook models of normal functioning and real time diagnosis and treatment of cases based on exhibited behaviour. For this a much simpler interface to the simulation model was required.

\section{The Simulation System}

The underlying models (figure 8 shows the calcium model) for the computer- based simulation were developed using Stella, a modelling package initially only available for Apple Mackintosh. These were used in conjunction with StellaStack software - a HyperCard stack adapted to provide a "user-friendly" interface for the Stella Models. This prototype was used to test the design of the system. However, a requirement was that this system be ported to a PC platform in order to enable larger trials to be conducted, the models were hard-coded in $\mathrm{C}++$ using 
the differential equations from the original Stella model. The simulations were supported by tutorial material written using Windows help files. Each of the models has switches in them to simulate nine different diseases and these switches produce predictable changes in various parameters that are commonly measured in medical laboratories. Administration of treatments can be simulated and the effects of the treatments on the different parameters monitored.

There are two interfaces to the model, one for controlling the model (see Figure 2), and the other to monitor the response of the model to the various interventions (see Figure 3 ). In addition to the model, the system consists of case material (such as presenting signs and symptoms, plus relevant medical history and an explanation of the case) and general tutorial information in the form of hypertext Windows Help files. The presenting case material and the tutorial information are available throughout the case, but the debrief material is only available after the model has run for a given period. This was to prevent the students' from looking at the answer before they had attempted to work it out for themselves.

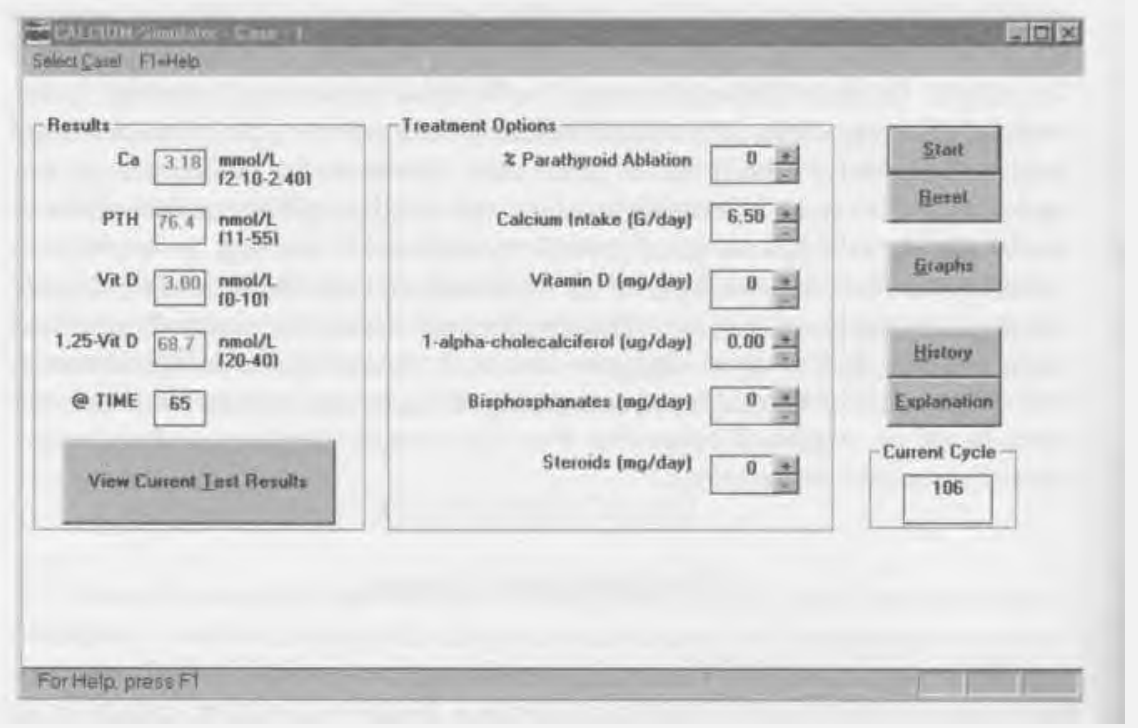

Figure 2. Interface showing the control of the model and the simulation of treatment options

Having developed a computer based simulation system, for helping students learn about metabolic disorders, the aim of this study was to test the hypotheses that the system adds value to the traditional lectures by fostering a deeper 
understanding of calcium homeostasis. In addition, it is hypothesised that this deeper understanding would be transferable to another body system.

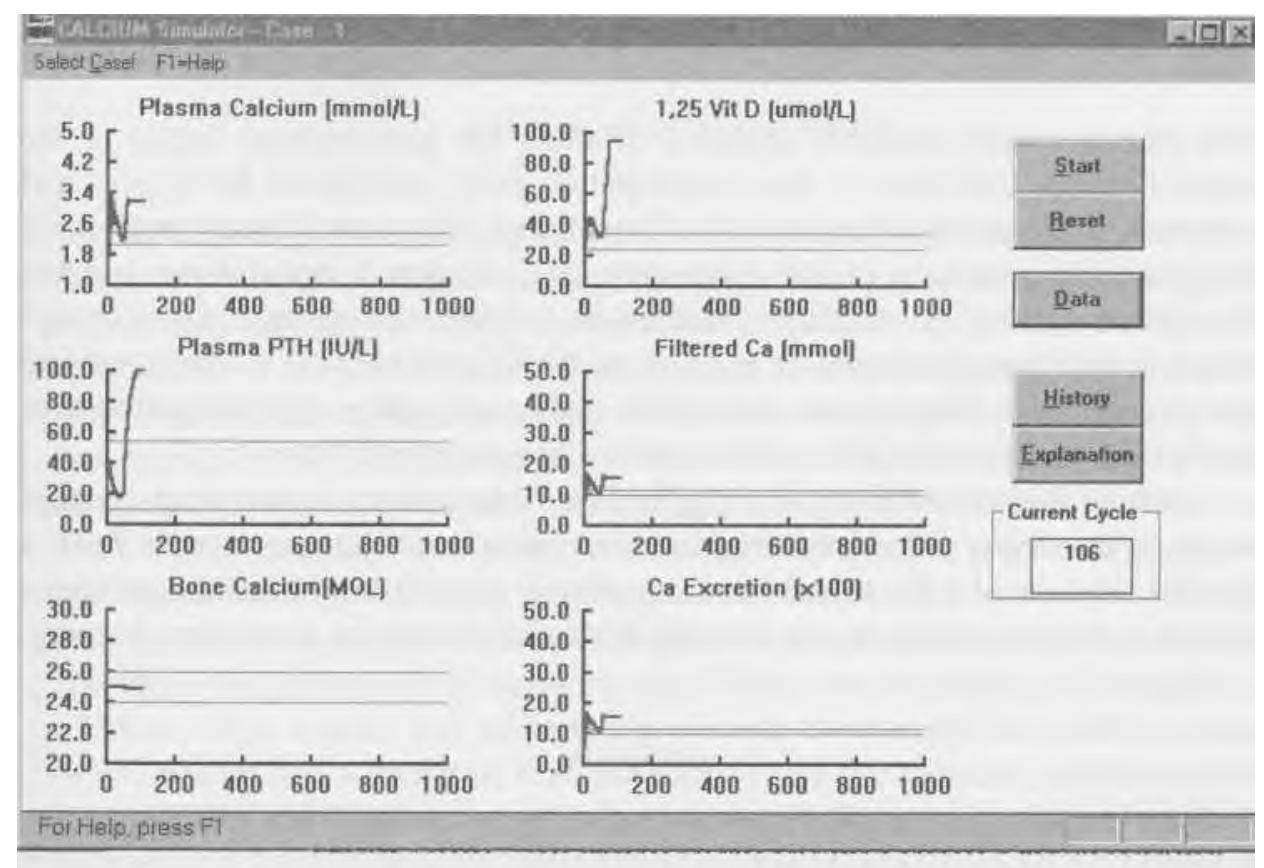

Figure 3. Interface for monitoring the output from the model

To assess whether there was learning attributable to the system, the investigation needed to demonstrate that students who used the system gained more than those who did not. The traditional, or accepted, method of assessment for medical students is Multiple Choice Questions (MCQs). However, as it was doubtful that MCQs alone would provide a sufficiently sensitive measure of learning, Concept Maps were also used as an additional measure of learning. In addition, the maps could be used to provide an insight into the learning process, through tracking belief change in association with actions performed on the simulation model. A third possibility investigated was that the maps themselves could affect the quality of the learning experience and therefore could be associated with learning gains in their own right. In other words, the process of constructing a Map would cause students to reflect upon and reason about the expected behaviour of the system and changes to normal functioning due to particular disease states. By testing their prior reasoning against the behaviour of the simulation, they might reason further and so deepen their understanding of the system further. 


\section{The Investigation}

\section{Design}

The students were randomly assigned to either the experimental Group or the control group. Condition 1, the experimental group, performed MCQ pre- and post-tests and used the system; while Condition 2, the control group, performed the pre- and post-tests in the same way as Condition 1 but did not use the simulation system. All students were asked to construct pre and post Concept Maps in addition to completing pre and post MCQ tests. The MCQ pre-test and the pre Concept Maps were undertaken one week before the simulation was performed and the post test and map were done one week later.

During the previous studies, students had been asked to construct concept maps, without any formal training but were provided with an example from a similar domain. As these had been completed without any apparent problems except with the content, it was decided that students did not need prior training.

\section{Subjects}

Groups of medical students rotate with the curriculum and spend four weeks on placement in the Chemical Pathology Department. Unfortunately, not all the students completed all the sessions, leaving gaps in the data. Where possible (without contaminating the data) students were retrospectively reassigned to different conditions so that as much of the data could be compared as possible. This resulted in groups of uneven size. A total of 132 students participated in the study. Of the 70 medical students who constructed two Concept Maps depicting the calcium system, 50 had used the calcium simulator before they constructed the second Concept Map. 64 Students constructed before and after Concept Maps depicting thyroid metabolism, of which 20 also used the thyroid simulator. There was overlap between the groups, in as much as some students produced four Concept maps and used both simulations.

\section{Procedure}

Students in the experimental condition answered pre-test MCQs, and constructed Concept Maps for both the calcium and the thyroid model. A week later they used the calcium simulation system, and in week three they used the 
thyroid system. The MCQs and Concept Maps were repeated in week four. Students in control condition did the same pre-test, but had a distracter activity - a laboratory visit in the second week. In the fourth week they did the post MCQs and the Concept Maps.

\section{Analysis of Concept Maps}

In this study, the marking scheme was required to distinguish between the flows or processes involved and the control of these processes. Accordingly, a scheme was developed whereby one mark was awarded for each correct process and one for each correct control mechanism, when compared to the expert's map. Because the maps produced by individual students were often idiosyncratic, any application of this scheme is to some extent subjective. With this in mind, marking was liberal and students were generally given the benefit of the doubt. Thus, where an entity was shown, without any flows half a mark was given, and where relationships between entities were shown but not named (or labelled) they were marked as correct. It was thought that the primary interest is in students knowing what is happening and why, rather than correct naming of processes. In addition, there was no way of knowing whether the student had omitted a label, or did not know the name of that relationship. As the calcium system involved more entities and controls than the thyroid system, the total number of marks for the calcium system was 14 , as opposed to the thyroid system for which the maximum score was 10 . Raw scores have been used except when a direct comparison was made between the two systems in which case the calcium scores were corrected, so both groups were marked out of 10 marks.

\section{Results}

All groups of students showed a gain on their second Concept Map. This was true irrespective of whether or not they used the relevant system.

Table 1. Concept Map scores for the Thyroid system

\begin{tabular}{|l|c|c|c|c|}
\hline & Number & $\begin{array}{r}\text { Pre maps } \\
(\max =10)\end{array}$ & $\begin{array}{c}\text { Post map (max } \\
=10)\end{array}$ & Gain \\
\hline Used Thyroid System & 20 & 6.28 & 7.43 & 1.15 \\
\hline Did not use Thyroid system & 44 & 6.68 & 7.016 & 0.33 \\
\hline Total & 64 & 6.56 & 7.13 & 0.58 \\
\hline
\end{tabular}


Table 2. Concept Map scores for the calcium system

\begin{tabular}{|l|c|c|c|c|}
\hline & Number & $\begin{array}{c}\text { Pre maps (max } \\
=14)\end{array}$ & $\begin{array}{c}\text { Post map (max } \\
=14)\end{array}$ & Gain \\
\hline Used Calcium System & 50 & 7.11 & 9.08 & 1.97 \\
\hline Did not use Calcium system & 20 & 5.775 & 8.1 & 2.325 \\
\hline Total & 70 & 6.73 & 8.8 & 2.07 \\
\hline
\end{tabular}

Thyroid system: 64 students did pre and post maps of the thyroid system. Of those, 20 went on to use the thyroid system, while 44 did not. The maximum possible score was 10. Mean score for pre maps was 6.55 and for post was 7.13. Difference between the two means using single factor ANNOVA was significant $p=0.016$ ( $f=5.92$ where Fcrit $=$ 3.916). Those who used the system appeared to show a higher learning gain than those who did not.

Similarly, 70 students did pre and post maps for the calcium system. Of those, 50 went on to use the calcium simulation, while 20 did not. The maps were scored out of 14 , and the mean score for the pre test was 6.73 and for the posttest 8.8 . With the calcium system there appeared to be larger gains amongst the group who did not use the simulation, but they did start from a lower initial knowledge.

Learning was demonstrated for all the groups who did pre and post maps, irrespective of whether they used the corresponding system or not. This gain appears to be greatest amongst those who did not use the calcium system, but as pointed out above those who did not use the simulations also appeared to have the lowest prior knowledge. It was noted that some students received lower scores on their post-map than they did on the preConcept Maps. There is no evidence that the students no longer believed that their first representation was correct - it is more likely that they were not sufficiently motivated to complete their maps accurately when repeating the task for the second time.

There were fourteen students who produced poor initial Concept Maps (students scoring less than half marks) but went on to produce comprehensive ones, showing an improvement of over $30 \%$. Of these, the worst cases initially produced a blank sheet of paper or one with just one or two unconnected concepts on it (see Figure 4). Of these 14 students who showed the lowest performance on the pre-Concept Maps, and highest learning gain, eight used the system while the other six did not. It is perhaps of interest to note that those students, who were least able to do the task, did not participate in the later sessions.

There were also groups of students who omitted certain entities such as the parathyroid glands, or failed to show how the regulation of the calcium was controlled (see Figure 5). 


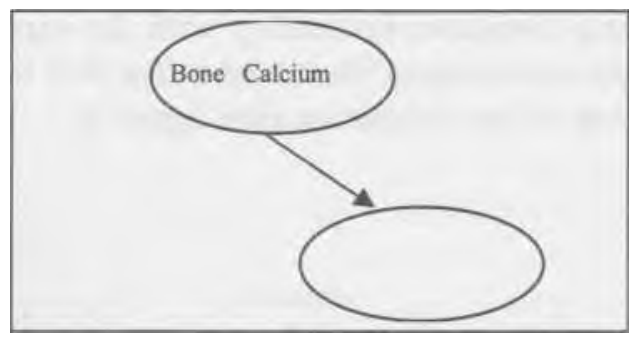

Figure 4. An example of a concept map showing low prior knowledge.

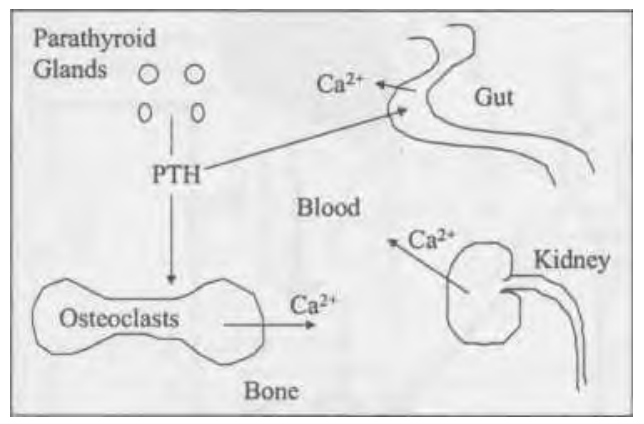

Figure 5. An example of a concept map showing incomplete representation, with little evidence of control mechanism.

The student who produced this calcium map however, went on to produce the comprehensive representation shown in Figure 6.

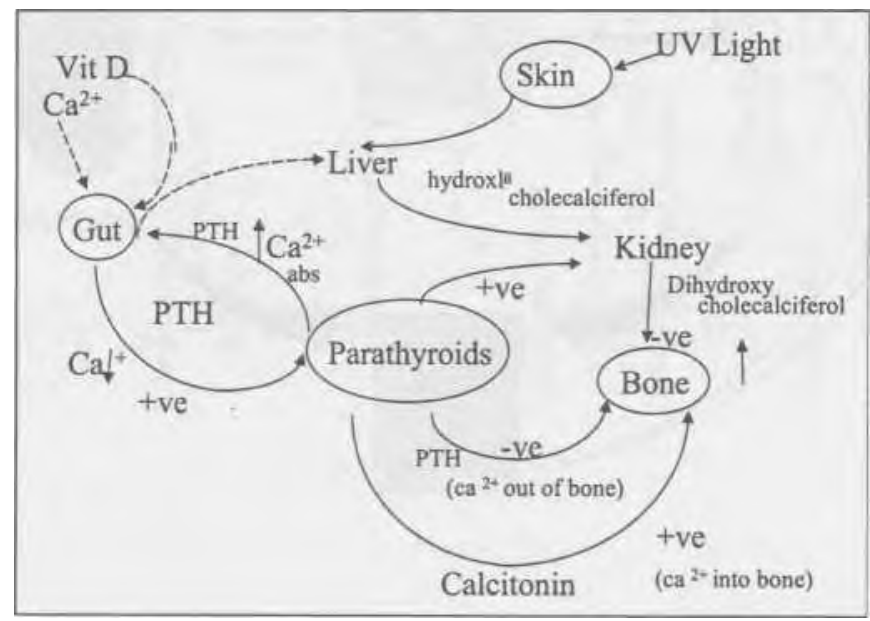

Figure 6. An example of a Concept Map showing comprehensive Knowledge. 
This concept map compares favourably with the expert model in figure 7 and includes the main sub-systems illustrated in the Full STELLA model which underpins the working of the simulation (see figure 8).

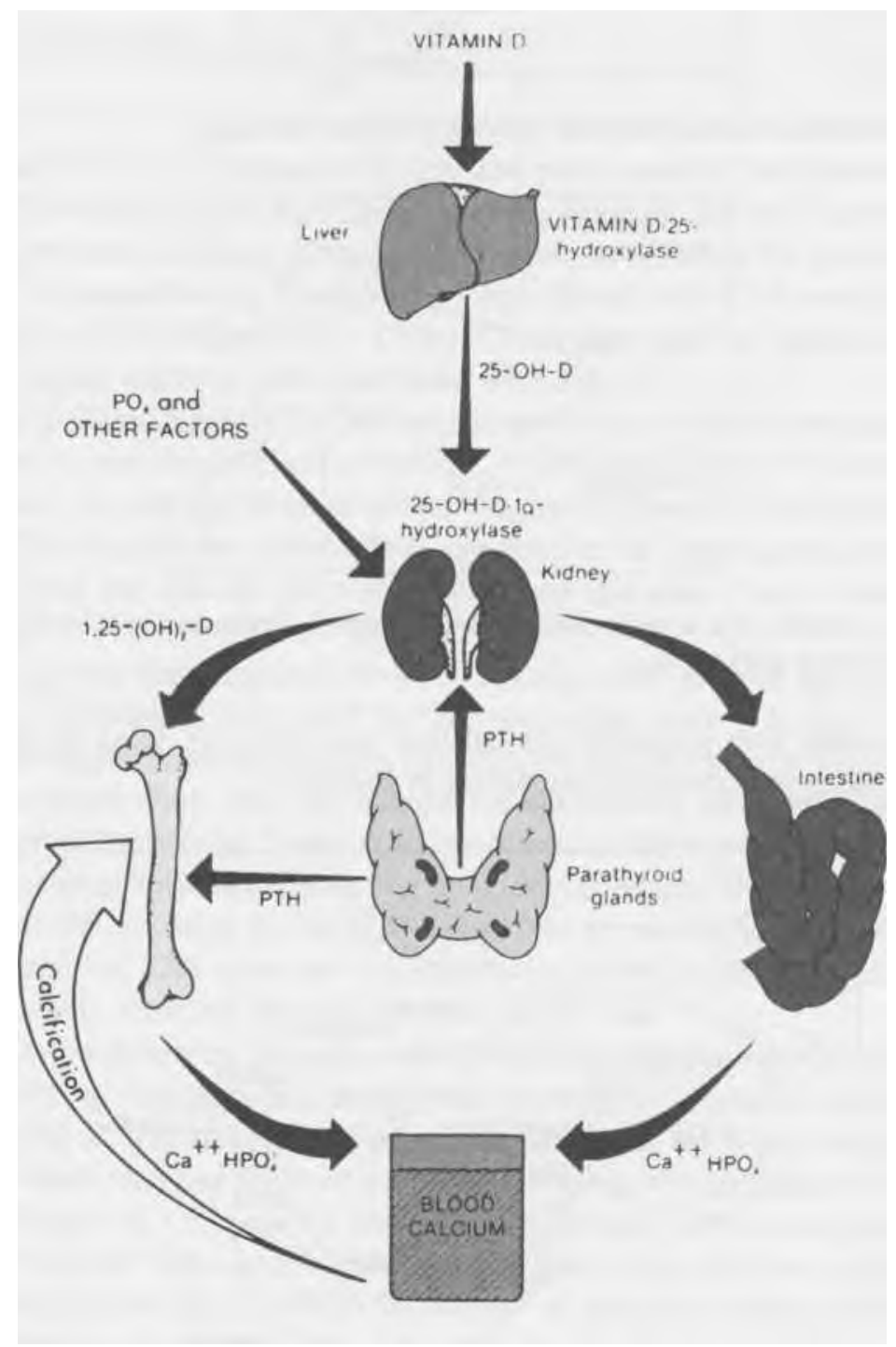

Figure 7. The expert model 


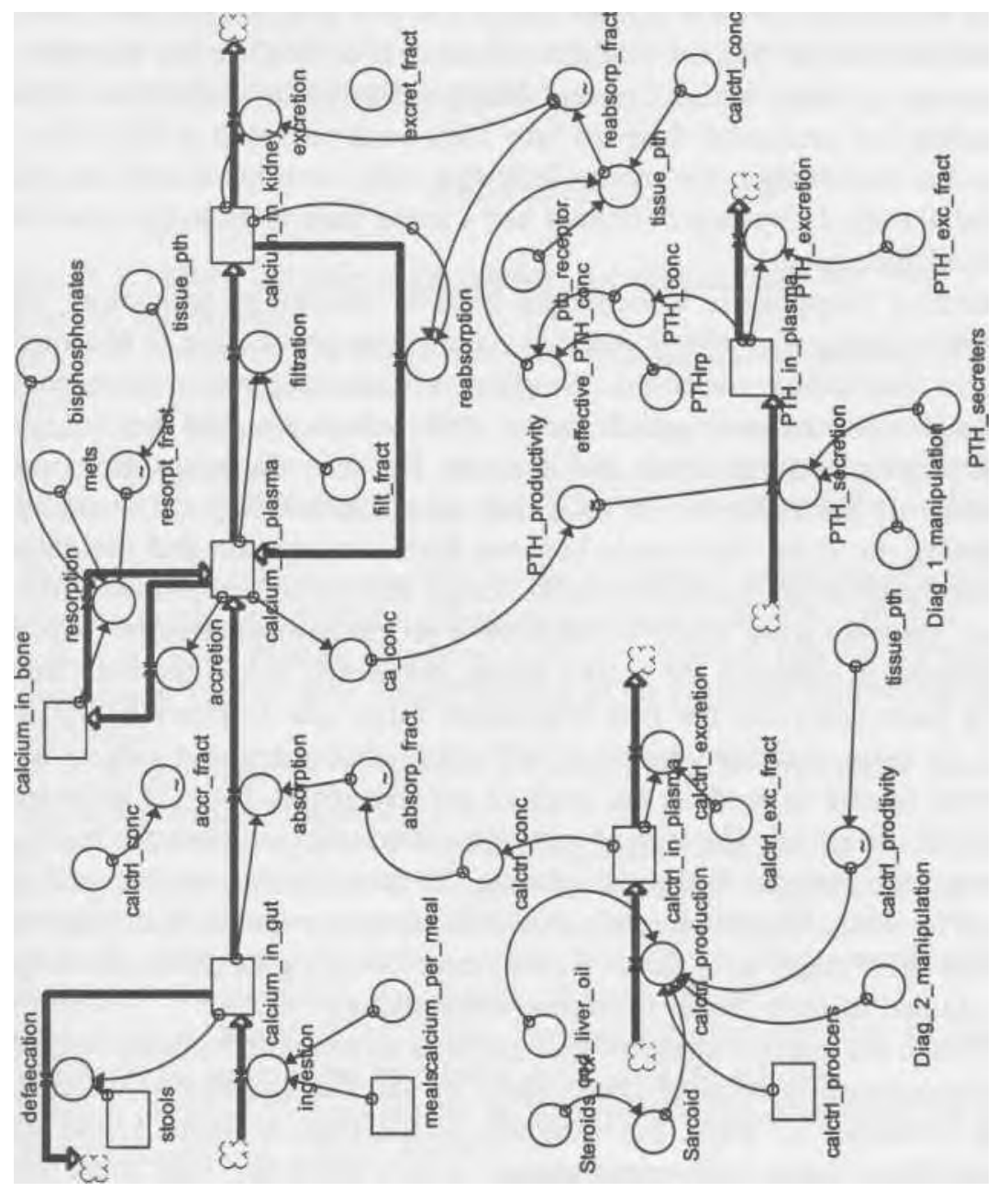

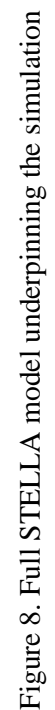




\section{Discussion}

Students improved their scores on repeating the Concept Maps, irrespectively of whether they used the system or not. Larger gains were found on the repeat calcium maps, than on the thyroid maps, but the largest gain was with those students who did not use the calcium system. It is difficult to see why this is the case, except perhaps initial Concept Maps indicated to students their lack of knowledge and prompted them to take their own remedial action. The lower their initial knowledge, the more likely this may have been, and the students who did not use the system definitely had a lower base knowledge, as evidenced by the calcium maps.

Concept Mapping is a technique used to encourage reflection. Though Concept Mapping was initially used as a performance measure, it obviously has a role in promoting reflection. Students appear to have a more complete knowledge when redrawing their maps, again whether or not they receive any intervening instruction about the domain. Because the maps were used as a measure of performance in acquiring an understanding of normal model functioning, no links were made between their construction and the diagnostic cases explored in the simulation tasks. In an attempt to make these links more explicit, students were asked to mark on a previously constructed map, where each disease is affecting the model during diagnosis. While the data from this has not been analysed, the first impression (after one session) is that it is of benefit to those students who have well established maps, but useless to those who were unable to produce the maps in the first place. There is an impression of cognitive overload. The task of using the software to complete the cases seems to occupy the students fully and relating the cases to the map as well proved difficult for some. On the plus side, however, there is evidence that some students modified their maps, as a direct consequence of trying to relate the diagnostic cases studied to their model of normal functioning.

Though the map the students were given as an example included only ellipses and interconnecting labelled lines, many of the maps produced differed from this in a number of ways. For example, in the map in figure 5, the students replaced the ellipses with organ shapes, which gives the map more concrete visual impact, which in turn makes it more memorable. Though many of the examples produced are similar to those found in standard medical textbooks, students constructed these for themselves in an active learning context, hence enabling reflection and more meaningful learning.

In conclusion, this study seems to have shown a definite role of priming in the use of Concept Maps. The maps seemed to focus students on the subject 
matter they were about to encounter in the simulation. Concept Maps also seemed to provide a good method of establishing prior knowledge, not only for the experimenter but also for the student. By reflecting on the gaps in the model as they attempted to draw the Concept Map, students were encouraged to reflect on what they knew about the metabolic system. This act of reflection seems to have been instrumental in bringing about learning gains for many students who did not use the simulation system. The detailed mechanisms by which drawing such maps impacts upon causal inferences made and, hence, problemsolving success is clearly worthy of further investigation.

\section{References}

Ausubel, D. P. (1968). Educational Psychology: A cognitive view. New York, NY: Holt, Rinehart and Winston.

Ausubel, D. P, Novak, J. D., \& Hanesian, H. (1978). Educational psychology: A cognitive view. New York: Rinehart and Winston.

Brown, A. L., \& Palincsar, A. S. (1989). Guided, Cooperative Learning and Individual knowledge Acquistion. In L. B. Resnick (Ed.), Knowing learning and Instruction: Essays in honor of Robert Glazer. (pp. 393-449). Lawrence Erlbaum Associates: Hillsdale, N.J.

Cox, R., \& Bma, P. (1995). Supporting the use of external representations in problem solving. Journal of Artificial Intelligence in Education. 6 (2/3), 239-302.

Derry, S. J. (1990). Flexible cognitive tools for problem solving instruction. In Anonymous. Bosten, MA: Annual meeting of the American Educational Research Association,

Gaines, B. R. and Shaw, M. L. G. (1995). Concept maps as hypermedia components. Int.J.Manmachine Studies 43131:323-361.

Glynn, S. (1997). Drawing Mental Models. The Science Teacher. Jan 97, 30-33 Heinze-Fry,

J. A., \& Novak, J. D. (1990). Concept Mapping brings long-term moverment towards meaningful learning. Science Education. 74 No 4. 461-472.

Johannsen, G., Rijnsdorp, J. E., \& Sage, A. P. (1983). Human System Interface Concerns in Support System Design. Automatica. 19 No 6. 595-603.

Jonassen, D. H. \& Marra, R. M. (1994). Concept mapping and other formalisms as Mindtools for representing knowledge. ALT - J. 2 No 1:50-56.

McAleese, R. (1994). A theoretical view on Concept Mapping. Association for Learning Technology Journal, 2 No 1. 38-48.

Novak, J. D., Gowin, D. B., \& Johansen, G. T. (1983). The use of Concept Mapping and knowledge vee mapping with junior high school science students. Science Education, 67 No 5. 625-645.

Novak, J. D., \& Gowin, D.B. (1984). Learning how to leam. New York: Cambridge University Press.

Paton, R. C. (1996). On an apparently simple modelling problem in biology. International Journal of Science Education. 18 (1), 55-64. 
Stewart, J., Van Kirk, J. \& Powell, R. M. (1979). Concept Maps: A Tool for use in Biology Teaching. American Biology Teacher 41.171-175

Stuart, H. A. (1985). Should Concept Maps be scored numerically? European Journal of Science Education. 7 No 1. 73-81.

Stuart, H. A. (1983). Studies in cognitive structure; Critique of assessments and representation techniques used by science education researchers. Science Ediucation Vol. 63, 395-405.

Suthers, D. D. (1999). Representational bias as guidance for learning interactions: a research agenda. In S. P. Lajoie \& M. Vivet (Eds.), Artificial Intelligence in Education: Proceedings of the 9th International Conference on Aritificial Intelligence in Education. Le Mans, France, July 1999. Amsterdam: IOS Press, pp. 121-128.

Wallace, J. D., \& Mintzes, J. J. (1990). The Concept Map as a research tool: Exploring conceptual change in biology. Journal of Research in Science Teaching. 27 No 11. 10331052.

West, L. H. T., Fensham, P. J. \& Garrard J. (1982). Final report: Describing the cognitive structure of undergraduate chemistry students. Monash University, Australia. 